\title{
A LASER POLAR MOTION EXPERIMENT
}

\author{
DAVID E. SMITH \\ Goddard Space Flight Center \\ PETER J. DUNN \\ Wolf Research and Development Corporation \\ and \\ RONALD KOLENKIEWICZ \\ Goddard Space Flight Center
}

\begin{abstract}
Summary. For three months in 1970, NASA, Goddard Space Flight Center conducted an experiment to try and detect the motion of the Earth's pole of rotation by laser ranging to an Earth satellite. Two laser tracking stations were used, one at Goddard Space Flight Center and one near Seneca in New York State, $408 \mathrm{~km}$ away and on the same meridian as the Goddard station. The objective of the experiment was to precisely measure the orbital inclination of the Beacon Explore $\mathrm{C}$ spacecraft, on a daily basis and with a resolution of a few hours, to a few hundredths of an arc second by the simultaneous tracking of the spacecraft as it passed between the two stations. During the experiment over fifty thousand range measurements on over two hundred revolutions of the satellite were obtained and on fifty occasions the satellite was simultaneously tracked at both stations. The noise accuracy of the tracking varied between 20 and $60 \mathrm{~cm}$ during most of the experiment and the orbital inclination obtained from the measurements showed a systematic change of about 25 arc sec, due primarily (about $80 \%$ ) to the gravitational perturbation of the orbit by the Sun and the Moon. Perturbations by solar radiation pressure and the solid-Earth tides appear to account for most of the remaining systematic changes in the inclination and from which it has been possible to derive a value of Love's number for the Earth as
\end{abstract}

$$
k_{2}=0.35 \pm 0.05
$$

A preliminary determination of the motion of the pole in the meridian containing the stations by subtracting the known perturbations (including tides) from the observed perturbation suggests that an accuracy of 3 to $5 \mathrm{~m}$ has been obtained, over periods of about $6 \mathrm{hr}$, during the latter part of the experiment. The experiment has also demonstrated that the present laser tracking systems are able to measure the chord distance between the two tracking stations with a repeatability of 25 to $30 \mathrm{~cm}$.

The present analysis of the data is directed towards an improved extraction of the gravitational perturbations and an improved determination of the Love number $k_{2}$.

A full account of the experiment and its results so far will be published elsewhere. 


\section{DISCUSSION}

R. J. Anderle: Have you estimated how the effects of Earth tides on the position of the two stations will effect the computed pole position?

D. E. Smith: I believe the stations are close enough for the tidal effects to be nearly equal.

P. Melchior: Over a distance of $400 \mathrm{~km}$, Earth tides can give a maximum change of length of something like $2 \times 10^{-8}$, so that is a maximum of $1 \mathrm{~cm}$.

P. Melchior: It is very important to point out how the tidal perturbation is connected with polar motion determination. From underground Earth tide measurements we derive $k=0.32 \pm 0.01$, you propose here $k=0.35 \pm 0.05$. There is practically no contradiction, but such a high value of $k$ would give too high a value for the Chandler period. Could you be satisfied with a $k$ value such as 0.32 ?

D. E. Smith: The 0.35 value is derived only from observational considerations without any tentative geophysical explanation. A value of 0.32 could be nearly as satisfactory but we need more observations.

$M$. Lefebvre: How long is the time span for each solution giving the length of the chord?

$D$. E. Smith: The longest single arc is $6 \mathrm{hr}$ but we estimate the chord distance from a number of these short arcs obtained over varying periods of time. The shortest period used here was about 10 days composed of 5 single 6 -hr arcs. The longest period was nearly 3 months and composed of about 20 short arcs.

Izuo Ozawa: Do I understand you to say that $k=0.3 \sim 0.4$ from your study? (1) Is there a phase difference between the observed tidal perturbations and the expected perturbations by $W_{2}$ ? (2) Did you consider the influence of the load tide caused by the oceanic tide? I think that the influence of the load tide is large and that it can be estimated.

D. E. Smith: We were unable to detect any phase lag between the calculated and observed perturbations of the orbit but this was probably due to the limited amount of data we have so far analyzed. We did not consider the effect of ocean loading. 